\title{
Enfoque actual en la rehabilitación de la escoliosis
}

\section{Current focus on scoliosis rehabilitation}

\author{
Naidelin Alonso González. ${ }^{1}$ Msc. Eugenio González Pérez. ${ }^{2}$ \& Lic. Juan Francisco Rojas \\ Puig. 3
}

\begin{abstract}
.
Idiopathic scoliosis consists of a threedimensional deformity of the spine, there is a lateral curvature of the spine or several, together with a vertebral rotation and a modification of the sagittal plane. An approach to scoliosis was performed; its definition, etiology and its therapeutic approach, specifically kinesiotherapy. The objective was to base the different methods that are in force, brief review of their authors, principles that govern them, their theoretical basis and application techniques.
\end{abstract}

Keywords: Scoliosis, physical therapy modalities, exercise techniques with

\section{Resumen.}

La escoliosis idiopática consiste en una deformidad tridimensional de la columna vertebral, existe una curvatura lateral del raquis o varias, junto a una rotación vertebral y una modificación del plano sagital. Se realizó un abordaje sobre la escoliosis; su definición, etiología y su abordaje terapéutico, en específico la cinesioterapia. El objetivo fue fundamentar los diferentes métodos que están vigentes, breve reseña de sus autores, principios que los rigen, su basamento teórico y técnicas de aplicación.

Palabras claves: Escoliosis, modalidades de terapia física, técnicas de ejercicio con

\footnotetext{
${ }^{1}$ Ciencias Médicas Mayabeque, Mayabeque, Cuba, nalfonso@infomed.sld.cu iD https://orcid.org/0000-00027353-0125

2 Ciencias Médicas Mayabeque, Mayabeque, Cuba, arceliagp@infomed.sld.cu 0002-7353-0125 ${ }^{3}$ Ciencias Médicas Mayabeque, Mayabeque, Cuba, jrojas@infomed.sld.cu iD https://orcid.org/0000-00027353-0126

https://orcid.org/0000-
} 
movements, exercise therapy, applied kinesiology, rehabilitation. movimientos, terapia por ejercicios, quinesiología aplicada, rehabilitación.

\section{Introducción.}

La unidad funcional del raquis se compone de dos vértebras, disco, articulación interfacetaria, ligamento intervertebral anterior y otro posterior, ligamento interespinoso y la musculatura. La columna en el plano frontal es prácticamente recta y cualquier desviación que cumpla ciertos requisitos, se llamará escoliosis 1, 2 .

La escoliosis se define como una deformidad de la columna vertebral en tres dimensiones, donde el plano frontal excede de diez grados y el desplazamiento lateral del cuerpo vertebral cruza la línea media y regularmente se acompaña de algún grado de rotación, es la afectación más agresiva y deformante que afecta al raquis.

La escoliosis no es un diagnóstico, ni una enfermedad, es la descripción de una alteración estructural, es un signo, es decir, una manifestación objetiva que se puede medir clínica y radiológicamente en la persona que la presenta; si en la medición no excede los diez grados, constituye una asimetría de la columna vertebral que no tiene significado clínico 3,4 .

Esta deformidad de la columna vertebral es un proceso complejo y dinámico y ocurre tanto en el plano sagital como en el frontal, principalmente en los segmentos toracolumbares. Las curvas en el adulto difieren principalmente en que son más rígidas que las de los niños o los adolescentes 5 .

En los adultos, además de representar una preocupación de tipo estético, se asocian a dolor y síntomas neurológicos, ocasionados por una combinación de fatiga muscular, desbalance del tronco, artropatía o artrosis de las facetas, por un proceso degenerativo discal; en los niños o adolescentes raramente manifiestan dolor, son descubrimientos de los padres al observar las espaldas de sus hijos, pero no por observación directa del portador de la escoliosis. En los pacientes que manifiestan dolor se requiere una valoración adicional para determinar la causa, principalmente en quienes refieren además sintomatología neurológica, o presentan una curva torácica izquierda 6,7 .

En más del $85 \%$ de los casos su causa es desconocida, se considera como patología mecánica del raquis, se concluye como un signo o síndrome de causa multifactorial, aunque el rol de factores genéticos se imbrica en los nuevos estudios 8 .

En el origen de la escoliosis, se encuentran tres categorías principales:

1. Neuromuscular: es el resultado de un desbalance muscular y la consecuente pérdida del control del tronco, se pueden encontrar curvas estructuradas y no estructuradas; estas últimas no tienen cambios anatómicos en su estructura ósea, son alteraciones funcionales relacionadas con vicios posturales de los adolescentes, discrepancias de la longitud de las extremidades o presencia de dolor. 
La deformidad ocurre en pacientes con padecimientos de origen neurológico o musculoesquelético, en el mielomenigocele, la distrofia muscular, la parálisis cerebral, o asimetría en la longitud de las extremidades pélvicas, la presencia de otros síntomas de la enfermedad subyacente ayuda a esclarecer el diagnóstico.

2. Congénita: es resultado de asimetría en el desarrollo de las vértebras, secundario a anomalías congénitas (hemivértebras, fallas de segmentación) se manifiesta en niños pequeños o antes de la adolescencia.

3. Idiopática: se define así una escoliosis donde no se encuentra una causa específica que explique el desarrollo de la deformidad. Regularmente es un diagnóstico de exclusión 9, 10.

Los signos característicos son: protrusión anterior de las costillas de un lado o de una escápula, diferencia en la altura de las caderas, hombros o pliegues glúteos, la cabeza no está centrada con respecto a las caderas; de pie, con los brazos colgando, el espacio entre un brazo $\mathrm{y}$ el tronco es mayor a un lado que al otro, y al agacharse hacia adelante con las piernas estiradas hasta que la espalda quede horizontal, un lado está más alto o abultado que el otro.

El diagnóstico de escoliosis se basa en los datos encontrados en una historia clínica completa, en la interpretación de maniobras clínicas dirigidas a valorar la movilización simétrica de la columna vertebral; se integran los resultados obtenidos en estudios auxiliares como los radiológicos simples, tomografía computarizada, resonancia magnética y estudio neuromuscular $11,12$.

Existen muchos métodos de ejercicios para la escoliosis, descritos en el siglo XIX y en la primera mitad del siglo XX, muchos ortopédicos y médicos en general e incluso algunos rehabilitadores no creen en la cinesiterapia, posiblemente basados en afirmaciones de libros clásicos o en su experiencia tras su inadecuada y simplista realización; sin embargo, la cinesiterapia, que es el tratamiento mediante el movimiento, es imprescindible para tratar todas las escoliosis independientemente de su etiología o severidad, en conjunción con la educación postural, el uso de órtesis y cirugía 13, 14.

Su aporte científico está dado porque se aborda de forma sintetizada y concreta los actuales métodos de rehabilitación en el tratamiento de la escoliosis, y su manejo por parte del fisioterapeuta sirve de referencia docente.

\section{Desarrollo}

La estrategia de búsqueda de información se realizó a través de los descriptores en ciencias de la salud, en Infomed; en las bases de datos de PubMed, LILACS, BIOMED, MEDLINE, ADOLEC, IBECS y EBSCO; con los descriptores: scoliosis, spinalcurvatures, musculoskeletaldiseases, bonediseases, spinaldiseases, intervertebral disc degeneration, intervertebral disc displacement; se redujo a otros descriptores como: physicaltherapymodalities, exercisemovementtechniques, exercisetherapy, kinesiologyapplied y rehabilitation. 
Se encontraron 12336 artículos y trabajos publicados en los últimos cinco años, se eliminaron contribuciones duplicadas, y se valoraron alrededor de 150 publicaciones, de las cuales se seleccionaron 40 para la bibliografía, que cumplían con los objetivos de la presente revisión.

Todos los métodos en el tratamiento de la escoliosis, se basan en tres principios directrices, documentados en la patomecánica de la escoliosis (tabla): facilitar la adecuada percepción postural al potenciar los músculos que mantengan la corrección, flexibilizar los segmentos cóncavos (trabajo isotónico concéntrico) y extensibilizar los músculos acortados (trabajo isotónico excéntrico) 15-17.

Tabla 1. Patomecánica de la escoliosis

\begin{tabular}{|c|c|}
\hline Lado cóncavo & Lado convexo \\
\hline La vértebra desciende & La vértebra asciende \\
\hline $\begin{array}{l}\text { Disminuye la altura del } \\
\text { hemicuerpo vertebral }\end{array}$ & $\begin{array}{l}\text { Aumenta la altura del } \\
\text { hemicuerpo vertebral }\end{array}$ \\
\hline $\begin{array}{l}\text { Reducida o ausencia de } \\
\text { cartílago epifisario }\end{array}$ & $\begin{array}{c}\text { Proliferación elevada de } \\
\text { cartílago epifisario }\end{array}$ \\
\hline $\begin{array}{l}\text { Disminución de la altura } \\
\text { del disco intervertebral }\end{array}$ & $\begin{array}{c}\text { Aumento de la altura del } \\
\text { disco intervertebral }\end{array}$ \\
\hline $\begin{array}{l}\text { Compresión de las carillas } \\
\text { articulares con formación } \\
\text { de osteofitos y sinostosis }\end{array}$ & $\begin{array}{l}\text { Descompresión de las } \\
\text { carillas articulares }\end{array}$ \\
\hline Canal vertebral estrechado & $\begin{array}{c}\text { Aumento del canal } \\
\text { vertebral }\end{array}$ \\
\hline $\begin{array}{c}\text { Las costillas se juntan y se } \\
\text { desplazan } \\
\text { anterolateralmente }\end{array}$ & $\begin{array}{c}\text { Las costillas se separan y } \\
\text { se desplazan en sentido } \\
\text { posterior (giba) }\end{array}$ \\
\hline $\begin{array}{l}\text { Retracción o fibrosis de los } \\
\text { músculos }\end{array}$ & $\begin{array}{l}\text { Elongación de los } \\
\text { músculos }\end{array}$ \\
\hline $\begin{array}{l}\text { Carencias nutricionales } \\
\text { con trastornos isquémicos } \\
\text { en hemidiscos y cartílagos }\end{array}$ & $\begin{array}{l}\text { Trastornos nutricionales } \\
\text { ocasionados por } \\
\text { distracción de los tejidos }\end{array}$ \\
\hline \multirow{2}{*}{$\begin{array}{l}\text { Las vértebras giran y } \\
\text { dirigen las apófisis } \\
\text { espinosas y los pedículos } \\
\text { hacia el lado cóncavo }\end{array}$} & $\begin{array}{c}\text { El cuerpo vertebral se } \\
\text { dirige hacia la convexidad }\end{array}$ \\
\hline & $\begin{array}{l}\text { Acuñamiento de la } \\
\text { vértebra ápex de la curva } \\
\text { con base en la convexidad }\end{array}$ \\
\hline
\end{tabular}

Fuente: International Research Society of Spinal Deformities (IRSSD) 
Existen diferentes métodos de cinesioterapia en la rehabilitación de la escoliosis; los métodos de Klapp, Niederhöffer, Schroth-Weiss y de Hans, son los de mayor divulgación, efectividad y aceptación. Menos empleados por su complejidad o poca divulgación en el país, están los métodos FED (elongación, desrotación y flexión) lateral de Cotrel, Sohier, Mézières, Souchard, Charrière y Roy, este último, se emplea más en el tratamiento de las algias vertebrales.

\section{Método de gateo de Klapp}

Klapp es un cirujano alemán de principios de siglo $\mathrm{XX}$, que basó su tratamiento para deformidades de columna vertebral en su observación de los animales, propugna que los animales cuadrúpedos, entre los que inicialmente se contaba el hombre, no tenían problemas de columna en su plano frontal, ya que las desviaciones laterales estando a cuatro patas eran casi nulas; al inicio no presentó su método como una terapéutica, sino más bien como gimnasia profiláctica

Figura 1. Método de gateo de Klapp

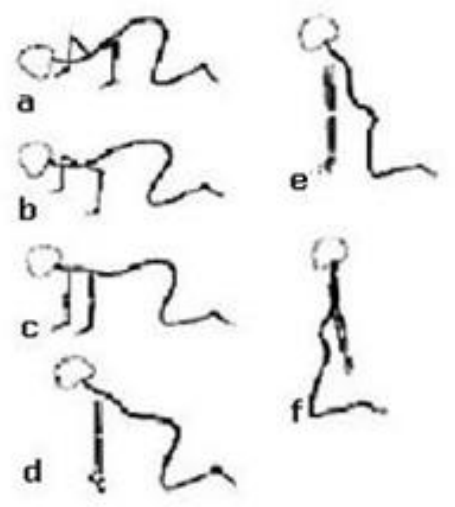
a. Posición baja: D1-D4
b. Posición semibaja: D6-D7
c. Posición horizontal: D8-D10
d. Posición semiergida: D11-L1
e. Posición ergida: L1-L3
(brazos hacia delante)
f. Posición invertida:L4-S1
(brazos hacia atras)
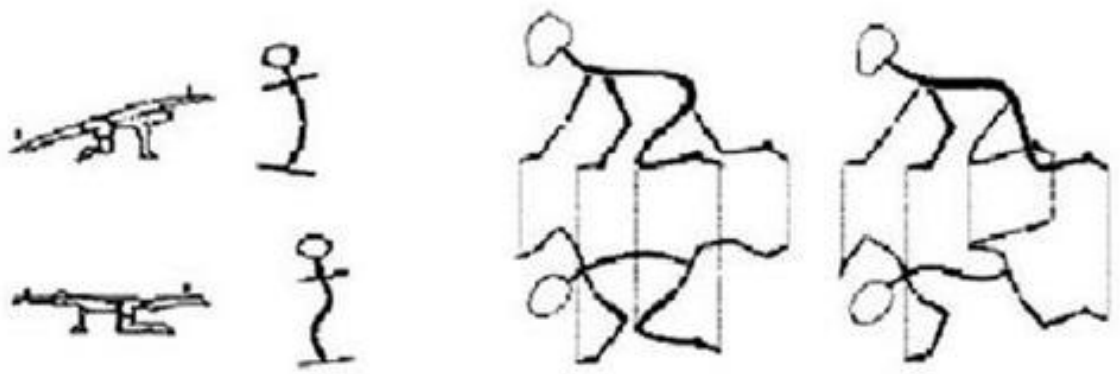

Fuente: Society on Scoliosis Orthopaedic and Rehabilitation Treatment (SOSORT)

Tiene como finalidad, desde una posición inicial, se descarga, se moviliza y con ello se corrige mediante la máxima tensión muscular, así se coloca al paciente dejando la columna suspendida de cuatro puntos de apoyo como si se tratara de una hamaca, la cual elimina la fuerza de la gravedad sobre la curva escoliótica. 
Las posiciones de gateo son cuatro que varían en función del segmento vertebral a movilizar; a estas posiciones iniciales se asocian dos tipos de ejercicios, la deambulación y los estiramientos, recomendaba su práctica al menos dos horas al día.

Se realizan ejercicios homolaterales de miembros superiores o miembros inferiores para curvas únicas (elevación de miembros contrarios a la deformidad) mientras que los ejercicios heterolaterales (miembro superior e inferior contrario) corrigen curvas dobles; también utiliza gateos en marcha curzada (curvas únicas) y marcha en ambladura u homóloga (curvas dobles).

Ejemplos de aplicación. Debilidades de la postura, escoliosis, espalda recta, cifosis, enfermedad de Scheuermann y enfermedad de Bechterew 18-20.

Método de musculación estática de VönNiederhöffer El método de Niederhöffer se basa en la potenciación de la musculatura transversa de la concavidad. Es un método analítico que fue muy revolucionario en su tiempo a finales del siglo XIX y principios del XX, pero que ignoraba por completo la educación postural.

Su finalidad es el fortalecimiento del tronco (cadena dorsal y ventral), para la elaboración de un corsé muscular; considera más importante la desviación lateral, e interviene analíticamente sobre grupos musculares trasversales (fig. 2).

Figura 2. Método de musculación estática de Vön Niederhöffer

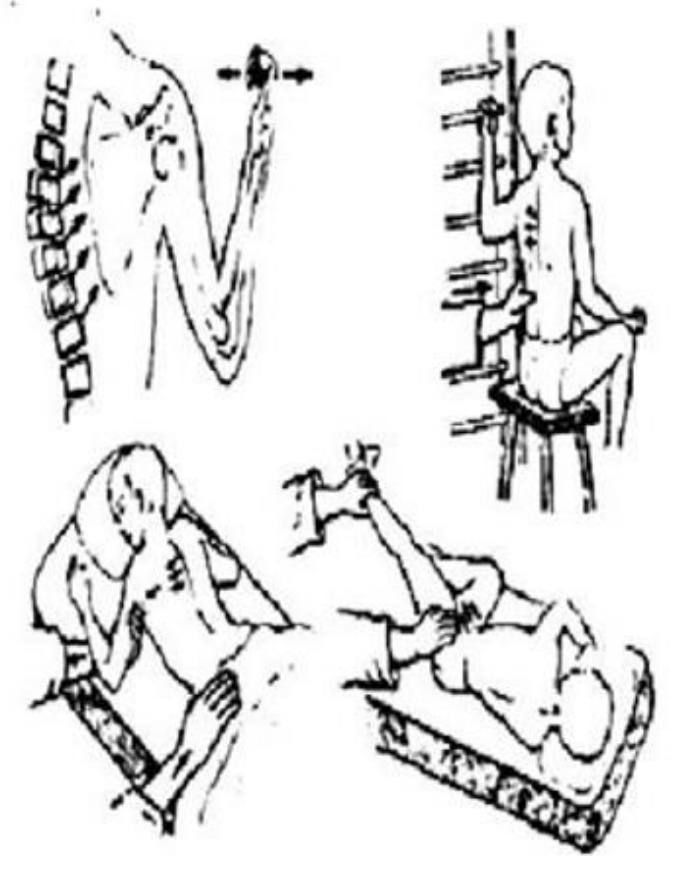

Fuente: Society on Scoliosis Orthopaedic and Rehabilitation Treatment (SOSORT) 
Corrige la curva por medio de contracciones isométricas de la musculatura trasversa de las cinturas en la concavidad. Su técnica se caracteriza por contracción lenta y progresiva, seguida por una fase de contracción isométrica, por último, retorno progresivo a la relajación.

Ejemplos de aplicación. Debilidades de la postura, escoliosis, espalda recta, cifosis, enfermedad de Scheuermann y enfermedad de Bechterew 21-22.

Método de KatharinaSchroth

Creado por la fisioterapeuta alemana Katharina Schroth en 1921, quien funda una clínica de rehabilitación de deformidades de columna en Meissen primero, y después de la II Guerra en Sobernheim. Su hija Christa Lehnert Schroth ha sido la gran divulgadora en Alemania; el Dr. Hans-Rudolf Weiss lo presentó más tarde a la comunidad científica.

Figura 3. Método de Katharina Schroth
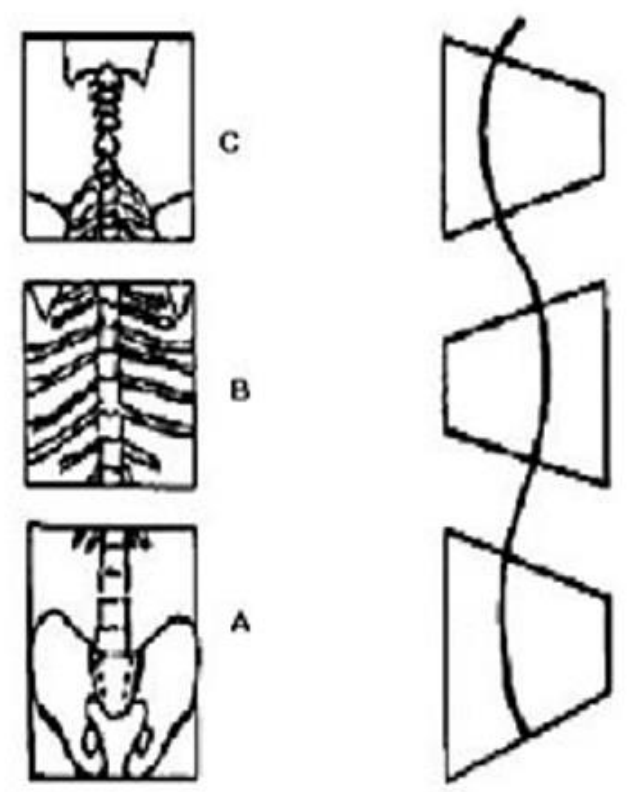

Fuente: Society on Scoliosis Orthopaedic and Rehabilitation Treatment (SOSORT)

Tratamiento tridimensional de la escoliosis, método de base sensomotor cinestésica que procura la corrección de la postura escoliótica, tridimensionalmente alterada y la corrección del patrón de respiración escoliótico. El paciente es entrenado de una forma individualizada por el fisioterapeuta a corregir su postura defectuosa mediante estímulos propioceptivos, exteroceptivos y el control de espejos.

Es una técnica respiratoria que busca la expansión del lado de la concavidad e introduce la conciencia postural con el mayor enderezamiento activo posible, para evitar en la vida cotidiana las posturas que agraven la escoliosis, su técnica de aplicación se desarrolla con. 
Ejercicios de suspensión: efectos predominantes sobre el aparato locomotor pasivo, estirando la musculatura acortada y aplanando la gibosidad costal.

Ejercicios de movilización: tiene por objetivo mantener, recuperar y mejorar la capacidad de movimiento de las articulaciones, sobre todo las de la columna vertebral, el tronco y la cintura escapular.

Ejercicios de modelación: ejercen un efecto desrotador sobre el tronco y la cintura torácica o escapular mediante la respiración desrotatoria que debe realizarse paralelamente.

Ejercicios de fortalecimiento y estiramiento: consisten en ejercicios musculares isométricos y de fuerza 23-25.

Método de Hans Mau o Polacou na nueva teoría de la etiología de la escoliosis idiopática, llamado el síndrome de las contracturas, se gesta en Alemania desde 1995, apoyada en la investigación primaria del Profesor Hans Mau, que se corrobora con diferentes estudios a partir de 2001 por los trabajos de los colaboradores TomaszKarski, Gardner, McMaster.

Esta teoría propone que la escoliosis es una deformación secundaria por la insuficiencia de aducción y en la rotación de la cadera derecha desde el nacimiento, necesaria para la marcha, la deformidad se inicia cuando el niño comienza a caminar por los movimientos compensatorios durante la marcha que provoca cambios en la pelvis y la columna vertebral.

Los síntomas son mínimos en el recién nacido, visiblemente en los bebés no tratados en la edad de 4 a 12 meses, y lo conforman: plagiocefalia, tortícolis, deformaciones de la pelvis, deformidades de los pies y la contractura de los músculos aductores de la cadera, en particular la derecha, en niños y adolescentes se observa al doblar la espalda, columna rígida y recta 26-30.

\section{Método Sohier}

Preconizó analizar y tratar todos los segmentos intervertebrales para que la corrección alcance progresivamente a toda la columna vertebral. Fue el precursor de los métodos quiroprácticos 31 .

\section{Método Mézières}

Ponderó la importancia de armonizar las curvaturas sagitales y las cadenas musculares para remodelar el cuerpo 32.

Método SouchardSouchard, colaborador de Mézières, basándose en sus principios, describió la rehabilitación postural global o método RPG. Asocia al programa de cinesiterapia respiratoria, la flexibilización y musculación para rectificar la lordosis 33, 34. 
Método Charrière y Roy Basan su método en la flexibilización de la curvatura escoliótica en el sentido de la corrección, así como en la potenciación muscular para que el paciente mantenga las posturas corregidas a lo largo de todo el día. Ellos defienden utilizar diferentes técnicas de distintos métodos y adaptarlos siempre a cada caso, así como la readaptación a través de la repetición cotidiana de los ejercicios hasta que se consiga la automatización de la corrección. Es indispensable la adhesión del sujeto y un riguroso protocolo.

Siempre habrá que flexibilizar, potenciar y rearmonizar propioceptivamente de forma individualizada y según cada fase del tratamiento; el tratamiento debe realizarse en equipo, con la elaboración de los objetivos terapéuticos por parte del médico, y una cinesioterapia personalizada, que debe ser enseñada y corregida por un fisioterapeuta 35, 36.

\section{Conclusiones.}

- La cinesioterapia de corrección de la escoliosis es un procedimiento imprescindible en su rehabilitación, con métodos bien establecidos de largo tiempo y con resultados muy satisfactorios independientemente de su etiología o severidad, en combinación con la educación postural, el uso de órtesis y la cirugía.

- Los mejores resultados se logran cuando existe una estrecha relación en el binomio paciente-fisioterapeuta y este último domina los diferentes métodos y aplica lo mejor de cada uno de ellos a cada uno de sus pacientes, teniendo en cuenta los hallazgos clínicos y radiográficos, tras las indicaciones del médico, es decir, tratamiento individualizado.

\section{Referencias bibliográficas.}

Kovacs FM, Mufraggi N, Gestoso M. Cómo es y cómo funciona la espalda. España: Fundación Kovacs; 2003 [citado 12 jul 2012]. Disponible en:http://www.espalda.org/divulgativa/como_es_funciona/comoes.asp

Vargas Sanabria M. Anatomía y exploración física de la columna cervical y torácica. MedLeg Costa Rica. 2012 [citado 5 jul 2012]; 29(2): 77-92. Disponible en:http://www.scielo.sa.cr/scielo.php?script=sci_arttext\&pid=S140900152012000200009\&lng=es

Vasiliadis ES, Grivas TB, Kaspiris A. Historical overview of spinal deformities in ancient Greece. Scoliosis. 2009 [citado 12 jul 2012]; 4(6). Disponible en:http://www.pubmedcentral.nih.gov/articlerender.fcgi?tool=pubmed\&pubmedid= 19243609 
Weiss HR. Spine Cor vs. Natural history explanation of the results obtained using a simple biomechanical model. Stud Health Technol Inform. 2008 [citado 12 jul 2012]; 140.Disponible en: http://www.ncbi.nlm.nih.gov/pubmed/18810014

Ryan EW, Ian AS, Xing Q, Matthew RW, James OS. Cobb angle progression in adolescent scoliosis begins at the intervertebral disc. Spine. 2009; 34(25): 2782-86.

Adams W. Lectures on the pathology and treatment of lateral and other forms of curvature of the spine. London: Churchill \& Sons; 2008.

Trobisch P, Suess O, Schwab F. Idiopathic scoliosis. DtschArztebl Int. 2010; 107(49): 87583.

Ogilvie J. Adolescent idiopathic scoliosis and genetic testing. CurrentOpinion in Pediatrics. 2010 [citado 12 jul 2012]; 22(1). Disponible en: http://www.ncbi.nlm.nih.gov/pubmed/19949338

Tello C. Escoliosis idiopática y discapacidad. Rev Asoc Argent OrtopTraumatol. 2010 [citado 28 jul 2012]; 75(1). Disponible en: http://www.scielo.org.ar/scielo.php?script=sci_arttext\&pid=S185274342010000100 $001 \& \operatorname{lng}=\mathrm{es}$

Grivas TB, Vasiliadis ES, Rodopoulos G. Aetiology of Idiopathic Scoliosis. What have we learned from school screening? Stud Health Technol Inform. 2008; 140: 240-44.

Smith JS, Shaffrey CI, Kuntz C. Classification systems for adolescent and adult scoliosis. Neurosurg. 2008; 63(3): 16-24.

Tejeda Barreras M. Escoliosis: concepto, etiología y clasificación. Ortho-tips.2011 [citado 12 jul 2012]; 7(2) Disponible en: http://www.medigraphic.com/pdfs/orthotips/ot2011/ot112d.pdf

Jiménez Ávila JM. Diagnóstico de la escoliosis. Ortho-tips. 2011 [citado 12 jul 2012]; Disponible en: http://www.medigraphic.com/pdfs/orthotips/ot-2011/ot112d.pdf

Mahaudens P, Banse X, Mousny M. Gait in adolescent idiopathic scoliosis: kinematics and electromyographic analysis. Eur Spine J. 2009; 18(4): 512-21.

Mahaudens P, Banse X, Mousny M. Influence of structural pelvic disorders during standing and walking in adolescents with idiopathic scoliosis. Spine J. 2009; 5(4): 427-33.

Negrini R. SOSORT guidelines: Orthopedic and Rehabilitation treatment of idiopathic scoliosis during growth. Scoliosis. 2012.[Citado 12 jul 2012]; 7(3). Disponible en: http://w09.biomedcentral.com/content/pdf/1748-7161-7-3.pdf 
Negrini S, Aulisa F, Ferrado C. Italian guidelines on rehabilitation treatment of adolescents with scoliosis or other spinal deformities. EuraMedicophys. 2008; 41(2):183-201.

Weiss HR. Best Practice in conservative scoliosis care. 3rd ed. Munich: Plaum Company; 2010.

Weiss HR. Spinal deformities rehabilitation-state of the art review. Scoliosis 2010 [citado 12 jul 2012]; 5(28). Disponible en: http://www.pubmedcentral.nih.gov/articlerender.fcgi?tool=pubmed\&pubmedid=211 84673

Martín Nogueras AM. Actuación fisioterápica en las escoliosis y en las actitudes escolióticas. Rev Asoc Argentina OrtopTraumatol. 2010; 75(1): 16-21.

Rigo M, QueraSalvá M, Villagrasa M, Ferrer M, Casas A. Effect of specific exercises on the sagittal profile of scoliotic spines. Scoliosis. 2007; 2 (1): 3-12.

Grivas TB, Vasiliadis ES, Rodopoulos G. The role of the intervertebral disc in correction of scoliotic curves. A theoretical model of idiopathic scoliosis pathogenesis. Stud Health Technol Inform. 2008; 140: 33-36.

Fong DY, Lee CF, Cheung KM. A meta-analysis of the clinical effectiveness of scoliosis school screening. Spine. 2010; 35(10): 1061-71.

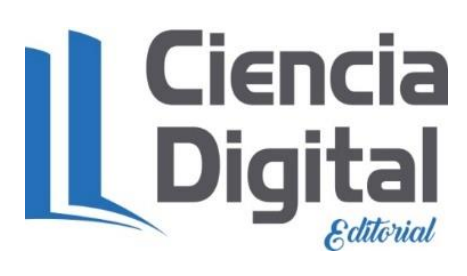




\section{PARA CITAR EL ARTÍCULO INDEXADO.}

Alonso González, N., González Pérez, E., \& Rojas Puig, J. F. (2019). Enfoque actual en la rehabilitación de la escoliosis. Anatomía Digital, 2(3), 6-17. https://doi.org/10.33262/anatomiadigital.v2i3.1083

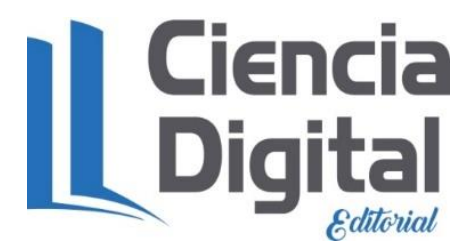

El artículo que se publica es de exclusiva responsabilidad de los autores y no necesariamente reflejan el pensamiento de la Revista Anatomía Digital.

El artículo queda en propiedad de la revista y, por tanto, su publicación parcial y/o total en otro medio tiene que ser autorizado por el director de la Revista Anatomía Digital.
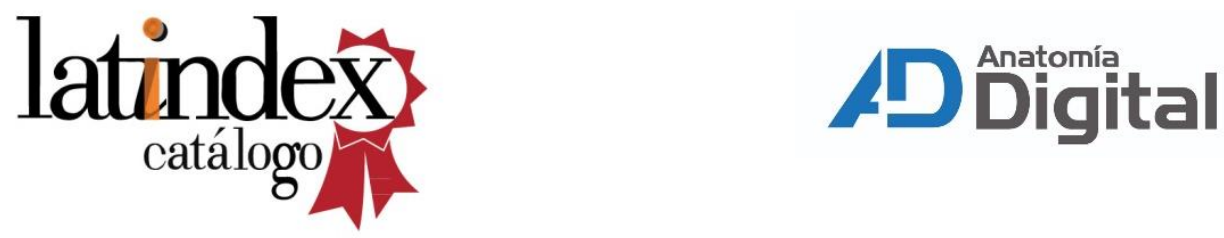